\title{
The indispensability of precollision kinematics in the visual perception of relative mass
}

\author{
SVERKER RUNESON and DANKERT VEDELER \\ Uppsala University, Uppsala, Sweden
}

\begin{abstract}
The visible kinematic pattern that occurs when objects collide contains information about the relative mass of the objects. Recently, Gilden and Proffitt (1989) have claimed that perceivers are limited to the use of simultaneous elemental cues and that therefore mass discrimination judgments are independent of whether the precollision epoch is visible or not. The present paper argues that their experimental results are irrelevant because crucial parameters were held constant, and that false conclusions were drawn because their data were not treated for what they are: threshold measurements obtained with the method of constant stimuli. Three experiments are reported, showing that a high level of performance is attainable only when the whole event is visible, and thus that the effective information is extended over time. In addition, modified PROBIT analysis was used to determine what information observers use when the precollision epoch is occluded. Analytically complex invariants, based on vector components in the abstract collision-axis system, proved to have the best fit to the data. Thus the elementaristic premises in Gilden and Proffitt's cue-heuristic model for relative mass perception are defeated.
\end{abstract}

Inherent in Michotte's work on the perception of causality (Michotte, 1946/1963; Thinès, Costall, \& Butterworth, 1991 ) is a significant refinement of the notion of event perception. In recent terminology, Michotte may have been the first to study dynamic events, events characterized by substantial, more or less abrupt, changes in the states of motion and with the perceptual emphasis on their causal (i.e., dynamic) aspects (Runeson, 1977/1983). This is in contrast to the majority of studies of event perception which concern kinematic events, relatively steady motions or changes that are taken to be perceived as such. In a large body of empirical work on collisions, Michotte demonstrated that, under appropriate kinematic conditions, it is not the kinematic properties of the event that one perceives. What is perceived is, instead, the type of causal relation (launching, entraining, etc.) that holds between the motions. Put simply, collisions are perceived as such, not as sequences of before- and after-motions.

More recently, it has been argued (Runeson, 1977/1983) that there is more to perceive in dynamic events than the mere fact that they are, for instance, collisions. As a background, it was shown that the kinematic pattern of a linear collision uniquely specifies the operative dynamic ("causal")

This research was supported by The Bank of Sweden Tercentenary Foundation (RJ) and The Swedish Council for Research in the Humanities and Social Sciences (HSFR). Parts of the present work were presented at the Sixth International Conference on Event Perception and Action, Amsterdam, The Netherlands, August 1991. Thanks are due Juri Allik for discussions on the processing of method of constant stimuli data, Dag Sörbom for statistical advice, and Geoffrey Bingham, Alan Costall, Emily Holmes, and William Mace for useful comments. Requests for reprints should be addressed to Sverker Runeson, Department of Psychology, Box 1854, S-75148 Uppsala, Sweden (e-mail: psysr@seudac21.earn). factors-namely, the ratio of the mass of the two objects and the effective damping due to the objects' material composition and shape. Further analyses have indicated that such kinematic specification of dynamics could be a general occurrence (the KSD principle), extending to animate events as well (Runeson \& Frykholm, 1983). Thus the KSD principle identifies a category of naturally available informational resources. ${ }^{1}$ It has thereby set the stage for a second phase of research-namely, empirical studies of whether perceivers can at all exploit such resources, the more or less idiosyncratic ways in which they may do so, the characteristics of the resulting performance, and its background in the nature of perceptual systems. Hence, the perceptual efficiency of KSD-type information has been the topic of several studies, mostly in animate events (e.g., Bingham, 1987, 1993; Runeson, in press; Runeson \& Frykholm, 1981, 1983), but also in mechanical events (e.g., Kaiser \& Proffitt, 1987; Todd \& Warren, 1982; Warren, Kim, \& Husney, 1987).

A challenge to the idea that dynamic properties can be visually perceived on the basis of kinematic information has recently been put forth by Gilden and Proffitt (1989; Gilden, 1991). In a study concerned with visual judgments of which of two colliding objects was heavier, they came to the conclusion "that the information available in the precollision epoch is not required"' (p. 382; cf. p. 377; reiterated in Gilden, 1991, p. 557). Their conclusion was based in part on theoretical considerations of the physics of two-dimensional collisions, from which they argued that the postcollision kinematics alone provide sufficient information about relative mass. It was also justified empirically by their Experiment 1, the results of which were claimed to show no degradation of performance when the precollision epoch was occluded; rather, it was concluded 
that performance was sometimes better when the initial kinematics could not be seen.

Special significance pertains to these conclusions since they form the basis for a model for relative-mass judgment proposed by Gilden and Proffitt (1989), and for an approach to "dynamical awareness" in general (Gilden, 1991; Proffitt \& Gilden, 1989). Essentially, their model denies the basic Gibsonian-ecological tenet that perception is based on analytically complex informative properties (invariants). Instead they claim that judgments must be made on the basis of elemental and simultaneously available cues (i.e., the velocities and directions of the postcollision trajectories), mediated by "some commonsense notions about how the world operates" (Gilden \& Proffitt, 1989, p. 374). It would be pointless to try to identify relevant invariants, thus to pursue the ecological approach, if perceptual access to the whole event could not be assumed. The purpose of the present study is therefore to critically examine the claim regarding the dispensability of precollision kinematics, both theoretically and empirically. In addition, evidence that could illuminate the invariants versus elementarism contrast will be sought.

On a theoretical level, Gilden and Proffitt's (1989) general claim is no doubt false. Although planar collisions are more complicated than most physical events studied in perception research, it is intuitively clear that, for instance, the object that moves faster after a collision need not be lighter. It might already have been moving at a good speed in about the same direction before impact or it might have been hit by a lighter but much faster moving object. It may also have a high component of velocity in the direction orthogonal to the collision axis that adds to its trajectory speed and alters its direction yet is independent of relative mass and momentum exchange (examples can be studied in Figure 5; the informational insufficiency of postcollision kinematics is illustrated in Figure 9). ${ }^{2}$ Thus, without access to the movements before collision, neither velocities nor directions are very informative about mass ratio. It is in the characteristics of the changes in the states of motion, incurred by the objects at impact, that there is information to specify which object is the heavier, or their relative mass (cf. Runeson, 1977/1983).

As it turns out, Gilden and Proffitt (1989; Gilden, 1991) have analyzed only a severely constrained type of collision: One object is always held stationary before collision. Furthermore, their proof is not correct for the constrained case either, unless it assumes that the perceiver has knowledge of the occurrence and nature of that constraint. Thus, the perceiver must have: (1) knowledge of the fact that the second object is initially stationary, and either (2) knowledge of the direction of the impinging object, or (3) knowledge of the orientation of the collision axis. Unfortunately, both (1) and (2) belong to the precollision epoch. As to item (3), the orientation of the collision axis could be known because it coincides with the direction of motion of the second object after collision-but only provided that it was stationary before collision, and known to be so. (This is because momentum can only be exchanged between colliding objects along the collision axis. If an object has no motion before impact, and hence no momentum, all the motion it can have afterward is bound to the collision axis.) Thus (3) falls back on assumption (1). Alternatively, (3) could be satisfied if the collision axis could be readily discerned from the spatial configuration of the two shapes at the moment of impact, yet item (1) would remain unsatisfied. In short, Gilden and Proffitt's proof lacks relevance for the general claims that they have made and is also insufficient for the special case analyzed. ${ }^{3}$

Even though inclusion of the precollision kinematics is in fact necessary for specification of relative mass, it might be the case that perceivers for some reason are unable to make use of informative properties defined over both epochs of the event. Gilden and Proffitt (1989) argue, in accordance with the cue-heuristic model they propose, that precollision cues are not utilized because it would require integrating information from distinct temporal intervals, by way of memory for the earlier motions. Thus, performance ought to be equally good or poor whether or not the precollision movements are visible, a prediction claimed to be borne out by the results of their Experiment 1 . However, an examination of Gilden and Proffitt's experimental design and data treatment reveals a number of shortcomings that invalidate the conclusion. In the following, their experiment will be critically discussed and three experiments will be reported that provide a more adequate assessment of the perceptual role of the precollision kinematics. The third experiment also identifies the (pseudo-) information that perceivers use in the aberrant condition that prevails when only the postcollision epoch is visible. Finally, it will be argued that the empirical facts refute the fundamental assumptions of the cue-heuristic approach put forth by Gilden and Proffitt (1989) and that instead an invariant-based approach is called for.

\section{CRITIQUE OF GILDEN AND PROFFITT'S (1989) EXPERIMENT 1}

\section{Incomplete Occlusion of Initial Kinematics}

The technique used by Gilden and Proffitt (1989) to test the contribution of the precollision kinematics was to overlay the display with a rectangular form that occluded all movement until the moment of contact between the two disks ("balls"), and then to remove it instantly so as to make the postcollision kinematics visible. Comparisons were made with the same collisions presented without the occluder. However, in the displays actually used in their experiment, the occluded condition was not fulfilled because only one of the objects was covered during the precollision epoch, while the other remained in view throughout the event. The justification for this omission seems to have been that the second object was stationary before collision, and hence, presumably, there were no kinematics to occlude. Although this might conform with a popular meaning of "motion," the null vector no less represents a state of motion. The immobility of the sec- 
ond object is an item of kinematic data as well, indeed one of crucial informational value, according to our discussion above. With the opportunity to observe the stationary object before impact, the observers therefore had access to information about half of the precollision parameters. Thus Gilden and Proffitt's experiment is not of a kind that could provide data in support of a negative conclusion concerning the role of precollision information. Minimally, a valid test would require full occlusion of both objects before impact.

\section{Representativeness of Conditions}

Gilden and Proffitt (1989) take care to point out (as did Runeson, 1977/1983, in criticizing Michotte's experiments) that it is necessary to have the experimental conditions sample the collision parameter space in a representative way if conclusions about perceptual ability are to be drawn. Thus there must be variation in all relevant circumstantial variables. If this is not observed, simple clues may suffice for correct selection of the heavier object within the confines of the particular experiment. Such results will not have relevance for the performance achievable under normally variable conditions. Furthermore, they will fail to bring in evidence any use of more advanced kinematic information that observers might be capable of.

However, Gilden and Proffitt's (1989) experiment falls short of satisfying this requirement. The precollision kinematics of two-dimensional collisions basically consist of four parameters that are free to vary, within limits, independently of each other: the velocity and the direction for each of the two objects. By keeping one object stationary, two of these parameters were held constant, which is a large departure from representativeness. Moreover, collision dynamics makes the case of a stationary second object special also in another respect-namely, that a change in the velocity of the impinging object only affects the temporal scale factor of the event. Thus the postcollision velocities will only change by the same proportion and the directions will be unaltered. It follows that, in this special case, variation in the impinging velocity makes no difference for the informative characteristics of the postcollision kinematics and therefore does not challenge perceptual capabilities the way it would if both objects moved before colliding.

In total, from the relevant four-dimensional parameter space, the experiment provided informationally relevant sampling on only one: Two angles of incidence relative to the stationary object were used. However, representativeness was further diminished by making this a betweensubject variable. In this way, the individual observer did not encounter any relevant variation in precollision kinematics. It is therefore also significant that the presentation of occluded collisions was interspersed with nonoccluded cases. Thus the observers got ample opportunities to tune into the standardized way that the objects moved and did not move, respectively, before collision and could rely on that in the occluded cases.

\section{Treatment of Results}

Gilden and Proffitt present the results of their Experiment 1 (1989, Figure 3, p. 376) with "percent correct" judgments as the dependent variable, plotted as a function of the logarithm of the mass ratio of the two objects. Although this procedure entails no formal error and seems unproblematic, it is actually very misleading and conceals the nature of the results. Thus, it has led the authors to mistake certain artifacts of the method of presentation for empirical results, and to draw some false conclusions on the basis of these artifacts. (The data presentations in Todd \& Warren, 1982, are subject to some of the same criticism.)

To clarify the matter, it should be observed that the purpose and structure of Gilden and Proffitt's (1989) experiment is in good accordance with the method of constant stimuli in classical psychophysics (see, e.g., Guilford, 1954, chap. 6). Objects are presented in pairs, and the observer's task is to give binary judgments as to which object in each pair appears to have the larger magnitude on the designated property. The independent variable is the actual physical difference or ratio and has an inherent equality point. ${ }^{4}$ The appropriate dependent variable is the frequency with which one of the objects is chosen, and it is expected to yield an ogive-shaped curve that crosses over from $0 \%$ to $100 \%$ somewhere along the independent variable. Thus, Gilden and Proffitt's experiment amounts to a threshold measurement of observers' acuity in distinguishing the relative mass of colliding objects. Performance should therefore be evaluated in two ways: the offset of the point of subjective equality (PSE) from the physical (the constant error) and the steepness of the crossover curve (the variable error; usually described inversely as the standard deviation, $S D$ ). The effects of other experimental variables (e.g., visible vs. occluded prekinematics) can be studied by comparing constant or variable errors, or generally by comparing crossover curves obtained for each such condition.

It follows that to get a clear picture of the results of Gilden and Proffitt's (1989) Experiment 1, their Figure 3 must be redrawn so as to show the frequency with which one of the objects is judged the heavier: The top-down inversion of one half of the graph must be undone. The difference between a regular threshold plot and a percentcorrect plot is illustrated in Figure 1. In such a replotting, the two curves exhibit continuity and an incomplete ogive character can be discerned, allowing a comparison of the two thresholds. By measuring more precisely from Gilden and Proffitt's Figure 3, the PSE, which ideally is at the $1: 1$ mass ratio, can be gauged to be about $1: 1.5$ in the normal condition and $1: 1.75$ when the impinging object is occluded before impact. Thus, if one assumes that the difference is statistically significant, the data show that performance in the occluded condition is consistently poorer than performance when the whole event is visible. The actual result of the experiment is therefore the opposite of what Gilden and Proffitt claimed to have 


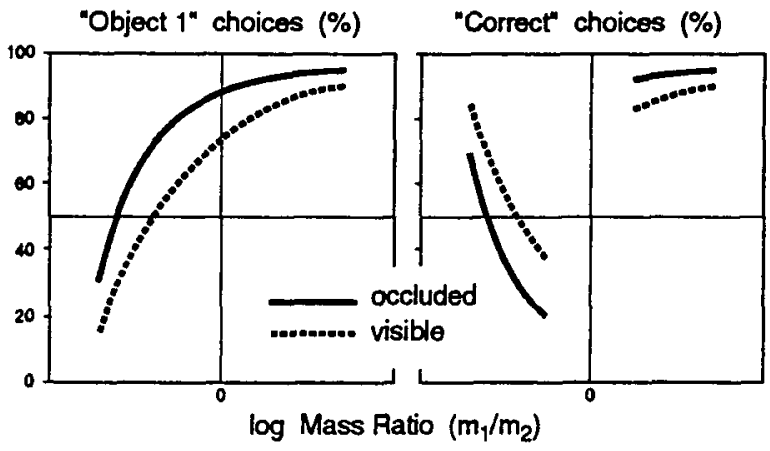

Figure 1. Comparison between a regular threshold plot (left) and a percent-correct plot of the kind used by Gilden and Proffitt (1989) (right). Both panels suggest the global features of their results; for the quantitative aspects, see Gilden and Proffitt's Figure 3. The disjunct percent-correct plot entails an inversion of the left-hand half of the panel and obscures the threshold character of the results. Regarded in terms of analysis of variance, the inversion makes a main effect of occlusion appear to be an interaction with mass ratio.

found. In terms of analysis of variance (ANOVA), there is indeed a main effect of the (incomplete) precollision occlusion, whereas no appreciable interaction between occlusion and mass ratio is in evidence.

Gilden and Proffitt (1989) go to some lengths in discussing various issues raised by the spurious interaction. In doing so, they come to claim that performance was sometimes better in the occluded condition, namely when the impinging object was the heavier $\left(m_{1}>m_{2}\right)$. This is a meaningless statement, since performance cannot be assessed separately for the two alternatives in a forcedchoice task. Assume that observers did not watch the displays at all and just chose Object 1 all the time. The logic employed by Gilden and Proffitt (see also Todd \& Warren, 1982) would then lead to the conclusion that discrimination was perfect in the conditions in which Object 1 was in fact the heavier, whereas it was totally absent when the mass difference was reversed. The truth is, of course, that no discrimination at all would be in evidence-just an extreme response bias. The elevated frequency of "correct" judgments for the occluded $m_{1}>m_{2}$ cases occurs because the PSE, and thus the whole transition region, is displaced somewhat farther out on the $m_{1}<m_{2}$ side. Hence it is just another symptom of the fact that performance is consistently poorer in the occluded condition.

\section{GENERAL METHOD}

To achieve a better empirical basis for conclusions concerning the role of precollision kinematics, a series of three experiments was conducted. The first was a refined version of Gilden and Proffitt's (1989) Experiment 1. A better controlled occlusion of the kinematics before impact was achieved, and the lack of representative variation in parameters was taken to the limit.

The second experiment went in the other direction concerning representativeness; we employed variation in several informationrelevant precollision parameters and in other kinematic aspects as well.
The purpose of the third experiment was to better assess the level of performance possible under normal viewing conditions, and to determine what (pseudo-)information observers are using when denied access to the precollision epoch. Thus, selected observers were employed and more practice was given. Normal and occluded cases were not mixed, and an even richer variability in experimental conditions was implemented.

\section{Simulation Technique}

Collisions were simulated and displayed by means of an in-house analog-computer simulation system that, unlike digital equipment, produces displays with infinite resolution and continuous motions. A digital computer (a Commodore 64 in the first two experiments, subsequently replaced by a 286-level MS-DOS system) was included in the system for controlling the experimental variables by means of multiplying D/A converters (configured as digitally set coefficient potentiometers), relays, and electronic switches. The digital computer was also used to control the sequence of events that made up the trials and the sessions: initial setup, displaying of collisions, recording of responses, administration of pauses, and so forth. In this way, the experimental sessions could be run without the continuous presence of an experimenter.

In analog simulation, motions are not computed or controlled as such. Instead, simulation occurs fundamentally at the level of forces that are integrated in real time to yield velocities. These are integrated once more to get displacements. Thus, the kinematic changes occurring in the collision were not achieved by precomputing postcollision motion vectors and switching over to them at the moment of impact, as in Gilden and Proffitt's (1989) and Todd and Warren's (1982) experiments. Instead, the impact event itself was dynamically simulated by summing the forces that arise while the objects are pushing against each other and feeding them to the integrator chains, thus effecting the appropriate changes of the states of motion. Two collision forces were involved. One was a springlaw function of the compression of the objects. This is a conservative force that builds up as the objects approach beyond the point of contact, and eventually pushes them apart again. The second force was a velocity-dependent dissipative force, representing internal friction (damping) in the objects. ${ }^{5}$

The technique employed entails that the simulation is realistic not only concerning the pre- and postcollision kinematics but also during the impact event itself. Thus, the impact unfolds over a finite interval, 130-160 msec in the first two experiments and 90-110 msec in the third. It also has an internal kinematics, consisting essentially of a compression of the objects' shapes in the collision dimension.

The lead-in motions were simulated natural-start motions: a constant-force acceleration that asymptotically levels off to constant velocity because of velocity-dependent dissipation. Previous research has shown that this kind of motion has a number of desirable perceptual properties (Runeson, 1974, 1975), the foremost being to appear constant throughout. This minimizes the conspicuousness of the start event so as to avoid interference with the perceiving of the collision itself. In most cases, the two objects were to have different velocities as they collided with each other. This was achieved by applying different driving forces and starting them simultaneously. In this way they also traveled different distances before impact. The time constant for the build-up of velocity was set to $.5 \mathrm{sec}$ and the approach movements took $1.5 \mathrm{sec}$, which means that the objects had reached $95 \%$ of their asymptotic velocity and acceleration had dropped to $5 \%$ of the initial value as the impact began. In view of the findings concerning the appearance of naturalstart motions, it can be assumed that the objects were perceptually in a steady state of motion when they collided with each other. ${ }^{6}$

The preceding description covers the simulation of one-dimensional collisions with motion on the collision axis only. Two-dimensional (planar) collisions were achieved by adding motion components in the orthogonal dimension. According to collision mechanics, these 


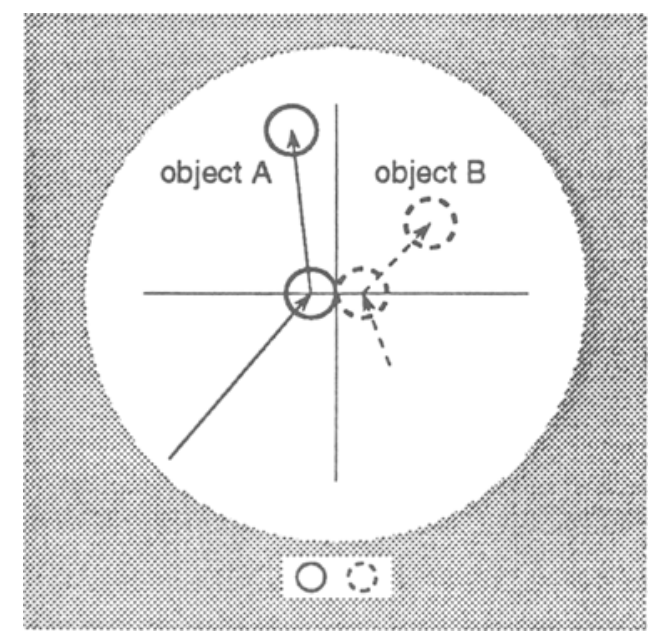

Figure 2. Arrangement and proportions of the display, with sample collision illustrated in canonical orientation. The two objects appeared in bright green against the dimly backlit circular viewing area and were distinguished by randomly giving one of them a broken outline. The small rectangular window is the response monitor display. Objects are shown at the moment of contact and at a unit time later. Arrows represent their velocity vectors before and after collision. Thin lines indicate the collision-axis system. Its dimensions are the collision axis (horizontal), on which all impact forces occur, and the sweep axis (vertical), along which each object maintains a constant velocity component throughout the event (axes not visible in the experimental displays). In the illustrated collision, common-mode velocity (explained under Experiment 2) is small and the impinging Object $A$ has a larger sweep component than does Object B. The mass of Object $A$ is $70 \%$ of Object B's. Refer to Figure 5 for the full variety of collisions used.

components can be different for the two objects but always remain constant across the impact event (Meriam, 1975, pp. 152-153). In reference to the latter fact they are here called the sweep components, occurring along the sweep axis (see Figure 2).

\section{Display Technique}

The collisions were displayed on a Tektronix 608 monitor oscilloscope with green high-intensity phosphor and then projected onto a ground-glass screen. The observers viewed the display from the opposite side through a large dual-lens Plexiglas collimator lens system ( $420 \mathrm{~mm}$ in diameter, $f=450 \mathrm{~mm}$ ) which allowed for free head movements in any direction without changing the effective angular properties of the display. Thus, in the following, screen measures in millimeters will be followed by the corresponding visual-angle measures in parentheses. The screen was dimly backlit by two neon indicator lamps to effectively mask the CRT afterglow traces. A black cardboard mask covered the screen, with a 140 -mm-diam $\left(17.7^{\circ}\right)$ circular opening in which the collision events were displayed. Another cardboard mask with a rectangular opening ( $200 \mathrm{~mm}$ wide, $100 \mathrm{~mm}$ high) was placed on the observer's side of the collimator to restrict viewing to the better portions of the lens.

The basic simulation, described above, produces four variable voltages representing the coordinates of the objects on the collision axis and the sweep axis. These signals were used to control the position of two circles, 13.5 -mm-diam $\left(1.7^{\circ}\right)$, in the display. Furthermore, a voltage representing the compression of the objects during impact was obtained and used to modulate the size of the circles in the collision dimension (up to $10 \%$ reduction). This small and brief deformation was only marginally noticeable, its main purpose being to avoid a conspicuous increase in brightness that would occur if the displayed contours were allowed to superimpose. The equipment allowed for mirror reversals on the collision and sweep axes and, in addition, rotation of the whole display in steps of $30^{\circ}$. The main features of the display are shown in Figure 2.

\section{Response Facilities}

The observers responded by pressing either of two microswitch buttons to indicate which object was perceived as heavier. To establish correspondence, one of the two objects in the display was rendered as a continuous outline circle while the other was distinguished by a broken outline. The allocation of the broken outline was determined randomly for each trial. The button tops were $18 \mathrm{~mm}$ in diameter and had a clearly felt ridge along the periphery, one of which was serrated to form a tactile counterpart to the brokenline circle in the display. To further reduce the possibility of mistakes, a response-monitoring display appeared below the circular display area. As soon as a button was pressed, a small luminous outline circle became visible; it was continuous or broken, depending on which button had been pressed. It remained visible for $2 \mathrm{sec}$, during which the observer could confirm the response and change it if required. The feedback provided in this way thus concerned the choice of button only, not the correctness of the response.

\section{EXPERIMENT 1}

The purpose of the first experiment was to compare the effects of complete and incomplete occlusion of precollision kinematics. It was essentially a replication of Gilden and Proffitt's (1989) Experiment 1, except that one, both, or neither of the objects was occluded.

\section{Method}

Gilden and Proffitt (1989) used two angles of incidence $\left(20^{\circ}\right.$ and $30^{\circ}$ ) and two velocities of the impinging object. As discussed above, neither of these variables contributed informationally relevant unpredictability. Since they also did not affect the results, they were held constant in the present experiment.

The incidence angle was set to $30^{\circ}$ relative to the stationary object, and the entire display was oriented to make the direction of approach horizontal. Right-left and up-down inversions of the display occurred randomly between trials. Following a natural start, the impinging object reached $51 \mathrm{~mm} / \mathrm{sec}(6.5 \% / \mathrm{sec})$ before it hit the stationary object. The approach movement took $1.5 \mathrm{sec}$ and began after both objects had been visible (except as called for by the occlusion conditions) at their starting positions for $.5 \mathrm{sec}$. A short beep was sounded the moment the movements began.

The simulated dissipative forces occurring during the impact phase were scaled to yield a restitution factor of .9 , representing about the lowest level of damping (highest "bounciness") achievable with real objects (cf. Warren et al., 1987). ${ }^{7}$

\section{Experimental Variables}

Mass ratio. Eight values of simulated ratio of mass between the stationary and the impinging object $\left(m_{B} / m_{A}\right)$ were used, covering the range from 1:4 to $4: 1$ in equal logarithmic steps. Thus the closestto-unity ratios were $1: 1.22$ and $1.22: 1$.

Occlusion. Occlusion of precollision kinematics did not involve the display of an occluding mask, as in Gilden and Proffitt's (1989) experiment. Instead the switch-on of CRT intensity for either or both circles was delayed until impact (more precisely, until the peak of the deformation). There were four occlusion conditions: No object, the impinging object, the stationary object, or both objects were occluded before impact. 
Each observer was presented with four replications of the $8 \times 4$ (mass ratio $x$ occlusion) factorial block, each containing all 32 experimental conditions in different random orders. Viewed as a method of constant stimuli measurement, mass ratio was the stimulus dimension and the threshold was measured under four different conditions of occlusion.

\section{Observers}

Fourteen observers ( 6 male and 8 female) were recruited from undergraduate psychology courses for participation in Experiments 1 and 2. Half of them received the experiments in reverse order. At least 2 days intervened between the two sessions. Observers were paid in proportion to their scores, calculated as the number of correct responses minus the number of incorrect responses. For each score point they received .50 Swedish crown; hence one Swedish crown (about \$.18) was at stake in each trial.

\section{Procedure}

The observers participated individually and were first informed generally about the study and instructed that for each collision shown he/she was to judge which object was the heavier. It was emphasized that the study concerned their ability to make fine distinctions in actual mass differences, rather than the subjective impressions they might have. They were also told that one or both of the objects would sometimes not be visible until the moment of impact, and they were told about the response facilities. Furthermore, they were informed that there was no time limit on responding and that once given, the response could be changed within $2 \mathrm{sec}$. After a response had been completed, the next trial followed automatically, with a few seconds' delay. Between the blocks of 32 trials, the apparatus called for a break by sounding short beeps at 4-sec intervals. In addition, the observers could initiate extra breaks by holding down both buttons in the interval preceding the next trial. Trials were resumed by pushing either button. The observers were encouraged to take breaks at any time they felt they were not able to perform at their best capacity.

\section{Results}

The judgments obtained from 2 of the 14 observers were highly erratic and have not been included. The combined results from the remaining 12 observers are presented in threshold-ogive form in Figure 3.

\section{Threshold Computations}

Standard computational procedures for the method of constant stimuli entail conversion of the obtained proportions to $z$ scores, followed by (weighted or unweighted) mean-square fitting of a straight line (Guilford, 1954, chap. 6). Unfortunately, the $z$ transformation does not give finite values for zero and unity proportions. Those data points either must be given zero weight, which means loss of information, or must be set to an arbitrary finite value, in which case an element of error is introduced.

In psychophysics, this problem is usually avoided by choosing a narrow range of variable stimuli, centered on the expected PSE value, and running a large number of replications. In this way, zero and unity proportions rarely occur. However, in the present study, the method of constant stimuli was applied to specific perceptual problems. Therefore, neither of the countermeasures mentioned above was feasible. The selection of stimulus values is dictated by the problems under study. To allow proper comparisons between experimental conditions, the range

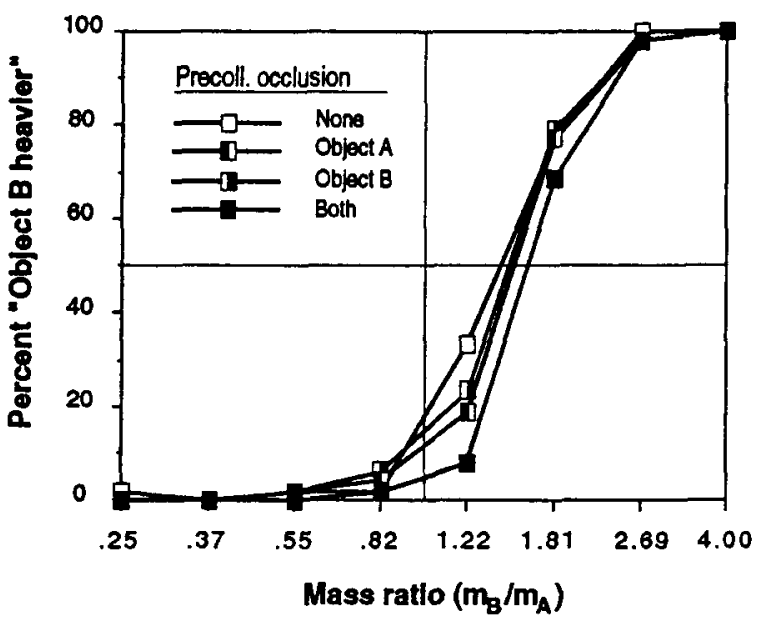

Figure 3. Results of Experiment 1. The impinging and the stationary object are indexed with $A$ and $B$, respectively.

of stimulus values must be kept wide in order to accommodate large differences in expected PSEs. Furthermore, the practical number of repetitions is limited by the need to determine thresholds under many different conditions (more so in the following experiments). It is therefore important to make good use of the information in every observation-that is, to estimate thresholds on the basis of as few run-throughs as possible.

The current data exhibit frequent occurrence of the extreme proportions; hence the standard methods were not suitable. Instead, thresholds were computed by means of nonlinear fitting of the cumulative normal function to nontransformed proportions. The iterative method used was similar to PROBIT analysis (described in more detail under Experiment 3). In this way, the information in the extreme proportions could be used to the full extent without arbitrary substitutions. Thresholds (PSEs) and difference limens $(S D \mathrm{~s})$ are presented in Table 1.

As raw data for statistical testing, individual thresholds were computed by fitting normal ogives to the individual sequences of 0 and 1 responses, using the same nonlinear function-fitting method as for the group thresholds. A twoway mixed-model factorial ANOVA was performed on the threshold measures, with occlusion as the fixed and observers as the random variable. The occlusion factor was not significant $(p>.1)$. Observers and the occlusion $\times$ observers interaction were significant $(p<.01)$; no system was apparent among the differences of the means, however.

Table 1

Mass-Ratio Thresholds Obtained in Experiment 1

\begin{tabular}{ccccc}
\hline & \multicolumn{4}{c}{ Objects Occluded in Precollision Epoch } \\
\cline { 2 - 5 } Threshold & None & Object A & Object B & Both \\
\hline PSE & .345 & .400 & .363 & .492 \\
SD & .284 & .289 & .317 & .231 \\
\hline
\end{tabular}

Note-Thresholds given as means (point of subjective equality, or PSE) and standard deviations $(S D)$ of fitted ogive, in units of ${ }^{\mathrm{e}} \log \left(m_{\mathrm{B}} / m_{\mathrm{A}}\right)$. 


\section{Discussion}

A comparison with Gilden and Proffitt's (1989) Figure 3 (cf. Figure 1 in the present paper) reveals a more distinct crossover character in the present results. Despite the improved implementation of precollision occlusion, it leads to less degradation of performance, if any at all. Thus, the outcome of the present experiment is possibly in better agreement with Gilden and Proffitt's (1989) contention that the precollision kinematics are not perceptually appreciated than were their own results.

However, the second main weakness of Gilden and Proffitt's (1989) experiment, the lack of relevant variation in the precollision parameters, occurred in the present experiment as well. For this reason, there was no need to (re)view the lead-in motions on each trial, and a disruption of performance in the occluded condition was not obligatory. In view of our previous comments on the informational role of the precollision kinematics, this is the most likely explanation for the small effect of occlusion in these experiments. The following experiments were designed to provide more relevant empirical data.

\section{EXPERIMENT 2}

\section{Method}

To allow representative conclusions concerning the effects of precollision occlusion, the lead-in motions, whether visible or $\propto$ cluded, must be varied unpredictably from trial to trial. Hence, parameters that were previously kept constant were turned into variables. The experiment was identical to Experiment 1, except for the differences described below.

If all possible precollision parameters were to be varied systematically, the number of conditions would be unmanageably large. Therefore, only parameters that were involved in determining the character of the collision itself were treated as experimental variables. Other kinematic parameters were varied randomly, thus reducing predictability further and improving ecological representativeness. Although the collisions always occurred at the center of the circular viewing area, neither the directions of the incoming objects nor the orientation of the collision axis could be known beforehand. The randomly varied parameters were the following: mirror reversal on the sweep axis, mirror reversal on the collision axis, and rotation of the entire display in steps of $30^{\circ}$ through $360^{\circ}$.

\section{Experimental Variables}

Mass ratio. Eight values of simulated ratio of mass between the impinged-upon and the impinging object $\left(m_{B} / m_{A}\right)$ were used, chosen as in Experiment 1 .

Common-mode velocity. The difference in velocity, as projected on the collision axis, between the two objects right before impact ( $V$ diff) was kept constant at $51 \mathrm{~mm} / \mathrm{sec}(6.5 \% / \mathrm{sec})$. Different precollision velocities were meanwhile achieved by adding a variable common-mode velocity component ( $V$ comm) to both. Vcomm was defined as zero when the objects moved at the same velocity (i.e., $V$ diff/2) symmetrically toward each other. As Vcomm increases, one object gets a higher velocity, thus becoming the impinging object (Object A), while the velocity of the other object gets correspondingly reduced, thus making it the impinged-upon object (Object B). At larger Vcomm values, Object B could be stationary, as in Experiment 1 , or be moving so as to be hit from behind. Two Vcomm values were used: $25 \%$ and $75 \%$ of $V$ diff. With the smalier value (labeled $V_{\text {comm }}$ small), the objects moved in opposite directions toward each other, at a 3:1 velocity ratio. With the larger value (Vcommlarge), they moved in the same direction, at a $5: 1$ velocity ratio, with Object A catching up and colliding with Object B from behind.

Sweeps. The collisions were made two-dimensional by adding motion components in the sweep dimension, orthogonal to the collision axis. There were two sweep conditions. In the first, both objects had equal sweep components of $23 \mathrm{~mm} / \mathrm{sec}(2.9 \% / \mathrm{sec})$ in the same direction. In the second, the sweep components had the same magnitude but were in opposing directions.

Occlusion. Either both or neither of the objects was occluded in the precollision epoch.

Each observer was presented with two replications of the $8 \times 2 \times 2 \times 2$ (mass ratio $\times V$ comm $\times$ sweep $\times$ occlusion) factorial block, each containing the 64 experimental conditions in a new random order.

\section{Observers}

The observers were the same as in Experiment 1 and were paid according to the same rule. Half of them participated in Experiment 2 first.

\section{Results}

Because of erratic judgments, 2 of the 14 observers (the same as in Experiment 1) were not included in the data treatment. A four-way mixed-model factorial ANOVA was performed on the individual threshold measures with Vcomm, sweep, and occlusion as fixed variables and observers as random. The following factors or interactions

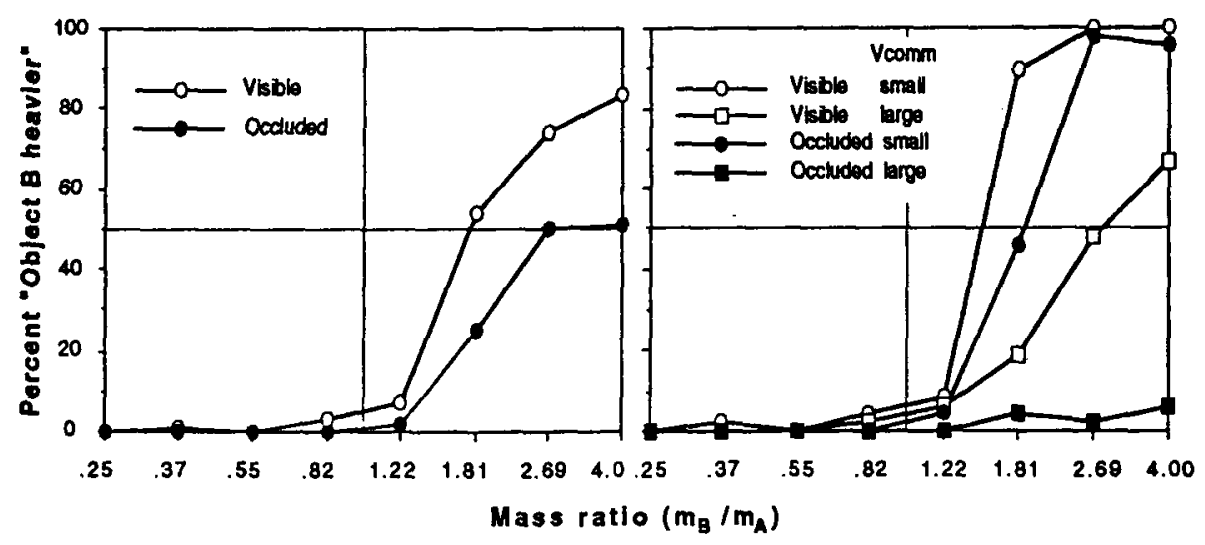

Figure 4. Results of Experiment 2. In the left panel, the data have been collapsed over the commonmode velocity (Vcomm) conditions. 
Table 2

Mass-Ratio Thresholds Obtained in Experiment 2

\begin{tabular}{|c|c|c|c|c|c|c|}
\hline \multirow[b]{3}{*}{ Threshold } & \multicolumn{3}{|c|}{ Normal View } & \multicolumn{3}{|c|}{ Occluded Precollision } \\
\hline & \multicolumn{2}{|c|}{$V_{\text {comm }}$} & \multirow[b]{2}{*}{ Combined } & \multicolumn{2}{|c|}{$V_{\text {comm }}$} & \multirow[b]{2}{*}{ Combined } \\
\hline & Small & Large & & Small & Large & \\
\hline PSE & .405 & 1.089 & .725 & .600 & $3.158 *$ & 1.181 \\
\hline$S D$ & .149 & .585 & .506 & .212 & $1.154 *$ & .631 \\
\hline
\end{tabular}

Note-Thresholds given as means (point of subjective equality, or PSE) and standard deviations $(S D)$ of fitted ogive, in units of ${ }^{e} \log \left(m_{\mathrm{B}} / m_{\mathrm{A}}\right)$. *By extrapolation; see Figure 4.

were significant $(p<.01)$ : Vcomm, occlusion, observers, $V$ comm $\times$ occlusion, $V$ comm $\times$ observers, and occlusion $X$ observers.

The results are presented in threshold-ogive form in Figure 4 and as threshold measures in Table 2. Unlike the results of Experiment 1, the results of Experiment 2 show a strong effect of occlusion. There was also an effect of common-mode velocity, which was much larger in the occluded condition. Observers differed somewhat in their susceptibility to the Vcomm and occlusion effects.

\section{Discussion}

For purposes of evaluating the role of precollision kinematics in relative mass discrimination, the results for the two Vcomm conditions should be combined to provide a more representative variation in the underlying conditions. This is illustrated in the left panel of Figure 4. The results are clear. Although there was a substantial constant error in the visible condition, in the occluded condition there was hardly any discrimination of relative mass at all within the studied range of mass ratios since choices of Object B only just reached the chance level. In addition, the variable errors are clearly larger with occlusion.

An examination of the right panel in Figure 4, where the $V$ comm conditions are separated, gives further insight into the consequences of being barred from the motions of the objects before collision. With a small commonmode velocity, a clean-shaped threshold was achieved, albeit not until Object B was almost twice as heavy as Object A. However, with a large Vcomm, Object A was consistently judged heavier throughout the range, even though Object B was up to four times heavier.

In summary, Experiment 2 showed that when only the postcollision kinematics are visible, performance is degraded so much that one could not properly speak of perception of relative mass any longer: The judgments were too far off the mark for that. Since they were nevertheless systematic, the question that arose was what property or properties of the postcollision displays might form the basis for those judgments. In particular, it would be interesting to test the explanatory potential of elemental cues, relating thus to the approach taken by Gilden and Proffitt (1989). The most obvious candidate was the relative speed of the objects; the faster moving object might appear to be the lighter. ${ }^{8}$ At $V$ comm $_{\text {small }}$, the threshold predicted on this basis would occur at an ${ }^{\mathrm{C}} \log$ mass ratio of .53 (1.70:1), which compared quite well with the ob- tained threshold, $.60(1.82: 1)$. For $V$ comm $_{\text {large }}$, the prediction fell outside the studied range (roughly at $7: 1$ ), as did the results. Thus, in Experiment 2, the results conformed quite well with the possibility that in the occluded condition the effective property was the relative postcollision speed.

Also in the visible condition, the main performance deficit consisted of a sizable offset in the thresholds due to the common-mode velocity component. Nonetheless, performance clearly surpassed the predictions from relative postcollision speed. The tentative conclusion was therefore that the observers had in fact taken advantage of the information in the precollision epoch, albeit not to the full extent. Since the observers differed significantly in this respect, it was possible that the conditions of the present experiment had not been suitable for revealing the level of performance that might actually be attainable.

\section{EXPERIMENT 3}

\section{Incomplete Invariants}

The present study is part of a project that seeks to characterize perceptual functioning and skill on a particular task in terms of a single proximal informative property. Perfect performance would occur if that property was a complete invariant - that is, a fully valid specifier of the distal property. Whenever performance differs systematically from perfection, this is, according to the working hypothesis, because the perceiver is relying on an incomplete invariant. This term stands for a proximal property that differs in some way from the complete invariant (Runeson, 1989a, 1989b; Runeson \& Costall, 1991). ${ }^{9}$ In the present context, any property defined exclusively over the postcollision epoch is bound to be an incomplete invariant, since an invariant that specifies relative mass under all conditions must draw on the precollision kinematics as well. As the crucial part of the approach, candidate incomplete invariants are to be suggested and evaluated as to their fit to performance data.

The large and systematic difference in performance between the normal and the occluded conditions suggests that observers use different information and thus that the approach should be applied separately to the two conditions. The occluded condition was focused in Experiment 3. Experiment 2 indicated that relative postcollision speed might be the property on which judgments were based in the occluded condition. However, the speed of either object is equal to the magnitude of the vector sum of its collision-axis and sweep-axis velocities. If, as in Experiment 2, both objects always have the same sweep velocity, there is thus a functional relation, and hence an experimental confounding, between speeds and collisionaxis velocities. The projections of the objects' motions on the collision axis therefore remained a viable alternative to the speeds.

To determine which of those properties might be perceptually more effective, it was necessary to break the covariation between them. It followed from what has been said above that this could be achieved by letting the two 
objects have different sweep-axis velocities and varying them relative to each other. A more representative variety of collisions was thus also achieved.

Pilot study. A design of this kind was first tried in a pilot study. The difference in sweep magnitude was made large enough so that it would either balance out or double the PSE offset due to $V$ comm $_{\text {small }}$ if relative postcollision speed was the perceptually effective property. The results did not conform with this expectation. Even in the occluded condition, the effect of the sweep variation was small and the thresholds fell closer to predictions based on the collision-axis velocities.

However, it is not clear how properties defined with reference to the collision axis could be perceptually accessed since there appears to be nothing in the postcollision motions themselves that could reveal its orientation. One possibility could be that the orientation of the collision axis is specified by the spatial configuration of the objects at the moment of contact between them: The collision axis is normal to the tangent at the contact point. In order to test this possibility, a mismatch (here called a glitch) was introduced in the alignment of the sweep components such that the displayed configuration of the two objects disagreed with the actual orientation of the collision axis.

\section{Method}

The experiment was identical to Experiment 2 except for the following.

\section{Experimental Conditions}

Glitch. Normally, the start positions of the sweep component motions were adjusted so that the centers of the two objects were aligned with each other, in passing, on the collision axis at the moment of peak impact. Without affecting the core simulation of the event, only the display of it, the desired glitch was achieved by starting one sweep from a little ahead and the other from an equal distance behind the normal positions. For the collision illustrated in Figure 2, this would mean that the trajectories of Object A were displaced downward while those of Object B were displaced an equal amount upward, or vice versa. Also, the objects were started a little closer to each other in the collision-axis dimension in order to close the gap that arises when the sweeps are out of alignment. In this way, the objects were displayed as contacting each other obliquely rather than straight during the impact, the center-to-center line deviating by about $30^{\circ}$ or $-30^{\circ}$ from the true collision axis. A no-glitch condition was also included. If velocity components projected on the skewed center-to-center line were the basis for the judgments, the PSE would be displaced by about $.25 \log$ units to either side of the no-glitch PSE (in the V comm $_{\text {small }}$ condition; the effect would be out of range in $V$ comm large). However, these were not predictions in the strict sense, since the glitch manipulation often led to oddities in the behavior of the objects in the first moment after impact. The glitch was thus an attempt to upset shape-based
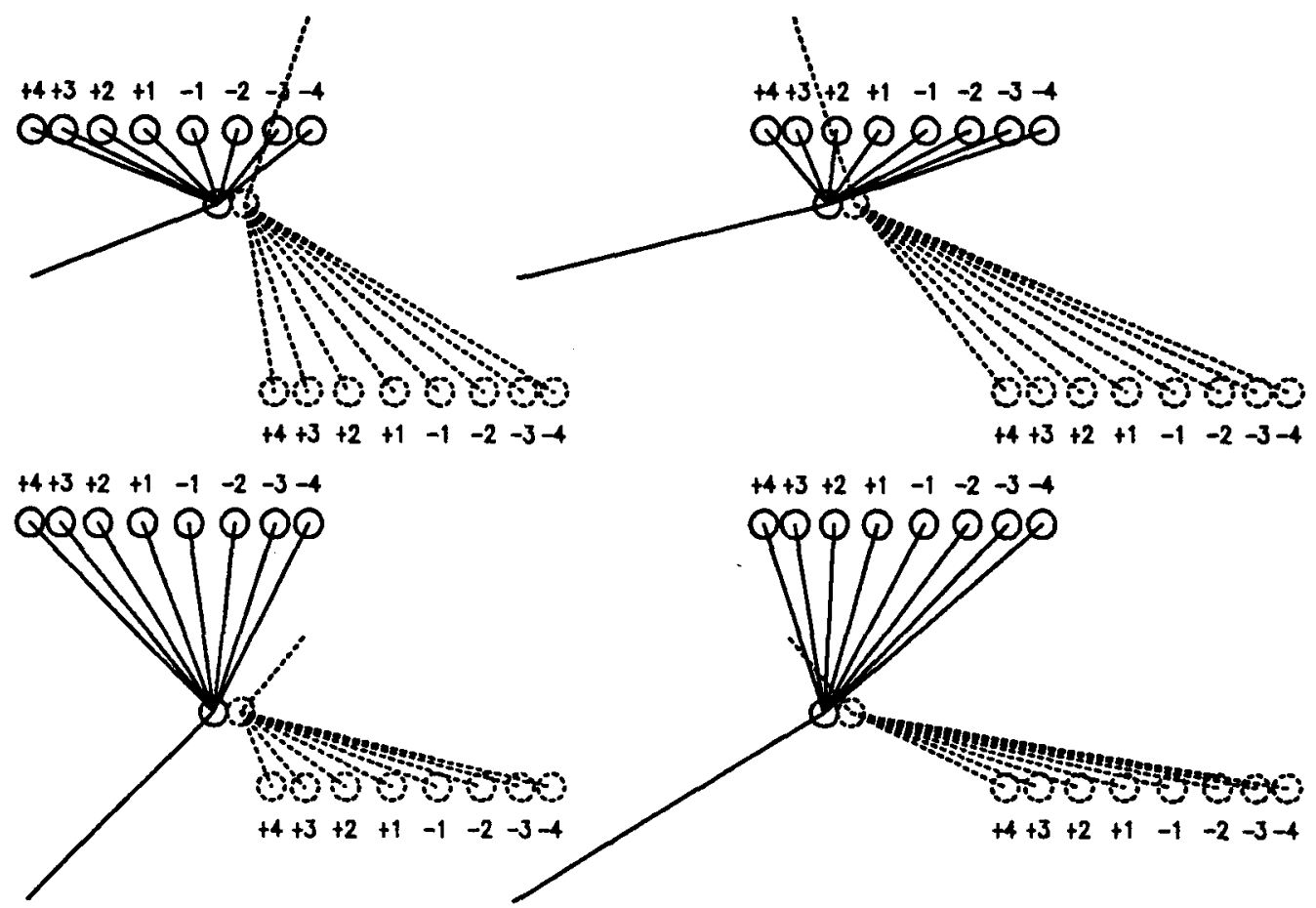

Figure 5. Kinematic diagrams of the collisions used in Experiment 3. Straight lines are velocity vectors with their head ends at the centers of the circles. Solid lines belong to the impinging objects (A), broken lines to the impingedupon objects (B). The collision axis is horizontal, the sweep axis vertical (orientation varied randomly around the clock in the experiment). Each group depicts eight collisions with identical lead-in motions and different postcollision motions due to different mass ratios. For pairing up the postcollision motions of the two objects, the mass-ratio conditions are indexed -4 through +4 (covering the 1:4 through 4:1 range used; equal mass would occur at index 0 ). In the top two groups, Object $B$ has the larger sweep component; below, it belongs to Object $A$. Left groups are for Vcomm small, right for Vcommberge. Glitch manipulations are not shown. 
specification of the collision axis rather than a provision of false information.

Sweep swapping. To break the functional relation between trajectory speeds and collision-axis velocities (see Figure 7), the sweep velocities for the two objects were always of unequal magnitude, either -14 or $35 \mathrm{~mm} / \mathrm{sec}\left(-1.8^{\circ}\right.$ or $\left.4.4^{\circ} / \mathrm{sec}\right)$. The faster sweep was assigned alternately to Object A or Object B, thus defining two sweep conditions.

Blocking and practice. Experiment 2 indicated that judging relative mass under normal and occluded conditions entails two different types of tasks. Thus interference between them might be reduced if the observers could focus on one task at a time and were given a chance to attune themselves to it before the test trials, especially to the awkward-seeming occluded condition. Therefore, occluded and visible cases were not mixed. Instead, observers received two visible and two occluded blocks in visual-occludedoccluded-visual order. The first of the occluded blocks was preceded by a practice session containing 64 occluded trials without glitch. During practice, the observers received feedback about which object was heavier, and each trial was repeated until the correct response was given. Because practice was given on the occluded condition only, the experiment was given some bias in favor of Gilden and Proffitt's (1989) contention that, overall, performance in the occluded condition should be as good as that in the normal condition.

In total, the design consisted of a random-order $8 \times 2 \times 2 \times 3$ (mass ratio $\times V$ comm $\times$ sweep $\times$ glitch) factorial block of 96 different collisions, administered four times to each observer (twice visible and twice occluded). The collisions are illustrated in Figure 5.

\section{Observers}

The basic tenet of Experiment 3 was that individual differences in performance on a certain task were due to observers' use of dif- ferent, more or less incomplete, invariants. Hence, it was not meaningful to search for an invariant that would explain a set of very divergent group data. The pilot study had indicated rather large differences between observers in both the visible and the occluded conditions. To achieve a more homogeneous group, 6 observers with prior experience from similar collision perception experiments were chosen. Four of them were students who had participated in one, differently designed, experiment and had proven to be medium or high scorers. The other 2 were the authors, who had gained extensive and varied experience in setting up several experiments in the project. The payment, awarded to the students only, was .50 Swedish crown (\$.09) per score point in the visual blocks and twice as much in the occluded blocks.

\section{Results}

The judgments obtained are presented in Figure 6, and the computed thresholds may be found in Table 3. A fiveway mixed-model factorial ANOVA was performed on the individual threshold measures, with Vcomm, sweep, glitch, and occlusion as fixed variables and observers as random. The following factors or interactions were significant $(p<.01)$ : Vcomm, occlusion, Vcomm $\times$ occlusion, and occlusion $\times$ observers. As in Experiment 2, the effect of Vcomm was much larger with occlusion. Furthermore, the observers differed slightly in the amount of decrement incurred in the occluded condition; but the differences did not set the 2 authors off from the students.

In comparison with performance in Experiment 2, performance in the visible condition was improved because of a substantial reduction in the effect of common-mode obj. A large sweep

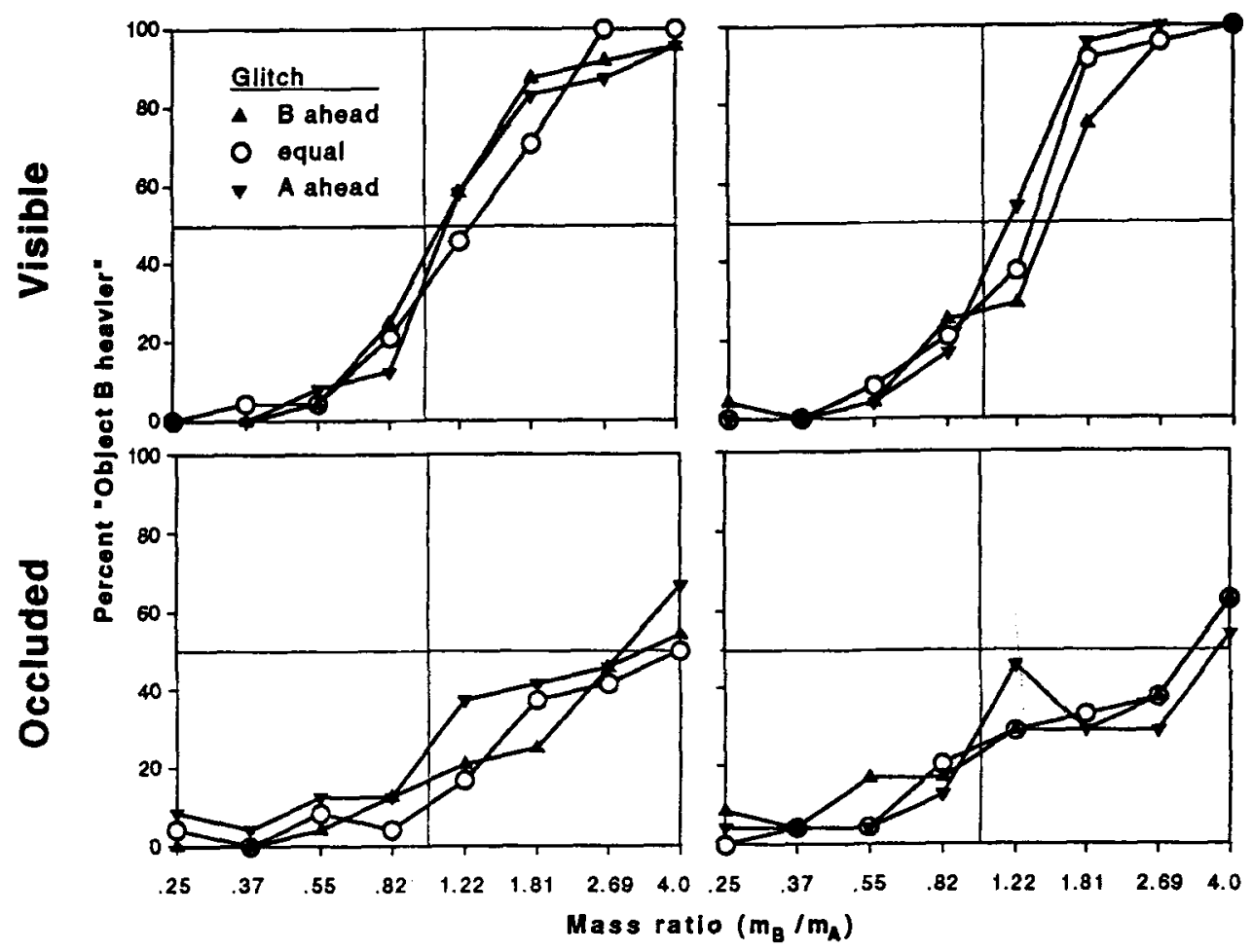

Figure 6. Results of Experiment 3. Data are pooled over the common-mode velocity (Vcomm) conditions. 
Table 3

Mass-Ratio Thresholds Obtained in Experiment 3

\begin{tabular}{ccccc}
\hline & \multicolumn{4}{c}{ Sweep Size } \\
\cline { 2 - 5 } Threshold & \multicolumn{2}{c}{ Normal View } & \multicolumn{2}{c}{ Occluded Precollision } \\
\cline { 2 - 3 } \cline { 5 - 6 } A $>$ B & B $>$ A & & A $>$ B & B $>$ A \\
\hline PSE & .197 & .186 & 1.143 & 1.198 \\
SD & .509 & .422 & 1.202 & 1.402 \\
\hline
\end{tabular}

Note-Thresholds given as means (point of subjective equality, or PSE) and standard deviations $(S D)$ of fitted ogive, in units of ${ }^{c} \log \left(m_{\mathrm{B}} / m_{\mathrm{A}}\right)$. Data pooled over the $V_{\text {comm }}$ and the glitch conditions.

velocity, both small and large. In contrast, performance in the occluded condition remained very poor, despite the extra practice that the observers had received. There was no appreciable effect of swapping the sweep magnitudes nor was there any systematic effect of the glitch manipulations.

\section{Discussion}

Through the separation of conditions and the use of somewhat experienced observers, Experiment 3 showed that the attainable level of performance on the binary mass-ratio judgment task was considerably higher than indicated by previous research. The PSE occurred already at a mass ratio of 1.2-that is, when the impinged-upon object had a $20 \%$ advantage in mass. (These figures have been corroborated by other experiments, without occlusion or glitch involved, in the present project.) This held for normal, fully visible, collisions only. Since no corresponding improvement occurred in the occluded condition, despite the supportive bias designed into the experiment, the evidence has been strengthened against Gilden and Proffitt's (1989) conclusion about the indifference of the precollision epoch.

The absence of an effect of swapping the sweep magnitudes proved that postcollision speed was not the perceptually effective property in the occluded condition. However, through the absence of a glitch effect, the support for the role of collision-axis velocity was unclear. The evidence entailed by the latter result was less conclusive, however, since the expectation of an effect was based on a certain assumption about how the collision axis might be specified and how it might be affected by the glitch manipulation.

In the visible condition, a glitch effect was not strongly expected, since the pre- and postcollision motions together were sufficient to specify the orientation of the collision axis; thus, the spatial configuration at impact was not of crucial importance. As to possible reasons for the absence of an effect in the occluded condition, it should be recalled that the glitch manipulation was not done with full technical finesse; for instance, the dimension of the compression of the objects was not reoriented. Also, the expected size of the effect might have been too small to be noticeable against the background of the indistinct thresholds that occurred with occlusion. Reports from observers indicated that some collisions looked unnatural. A simple follow-up confirmed that the glitch might be detected as such in some combinations of sweep swap and glitch direction. In these conditions, the objects overlapped briefly to some extent as they continued with their postcollision motions. These observations, combined with the fact that the glitch and no-glitch conditions yielded very similar results, suggest that the glitch manipulation was inefficient in upsetting the specification of the orientation of the collision axis-if one assumes that the information used by observers is related to the collision-axis system (see below).

\section{Evaluation of Incomplete Invariants}

The goodness-of-fit measures associated with regression of judgments on candidate incomplete invariants could provide a more sophisticated evaluation of their relative merits. However, since the judgments of mass ratio are not quantitative, ordinary regression analysis is not possible (cf. Burton \& Turvey, 1990, for a related case in which it is). Instead, "qualitative-response models" (Amemiya, 1981) fit the present needs. Because of its affinity with psychophysical threshold methods (Finney, 1971, pp. 38-42), the PROBIT method was chosen. In brief, the PROBIT method performs regression analysis between the observed probability (i.e., relative frequency) of the binary response and the independent variable. As the psychometric function, a cumulative normal curve is fitted instead of a straight line. The mean and standard deviation of the ogive are varied in an iterative search for a minimal loss value (defined as the negative of the loglikelihood function).

Error compensation. For the present purpose, a PROBIT analysis program was developed that used the simplex-type of iteration (written in Microsoft Professional Basic; the simplex routine was adapted from Press, Flannery, Teukolsky, \& Vetterling, 1986). It was found necessary to include a compensation for response errors, assuming that with some constant probability, $p_{\mathrm{err}}$, the recorded response would be the opposite of that which the observer intended. This error rate would subsume mistakes about which object had the broken outline, pushing the wrong button, technical malfunction (of which there was no indication though), and similar errors. The PROBIT method entails computation of loss on the basis of each individual recorded response $\left(p_{\text {resp }}=0\right.$ or $p_{\text {resp }}=1$ ), each one weighted according to the expected response frequency at that point on the independent variable. Thus, if the normal ogive describes the psychometric function, and $p_{\mathrm{ogi}}$ is its value at the current point, then the expected recorded frequency $p_{\exp }$ must also depend on perr. The straightforward way to take this into account is to take the expected probability of a " 1 " recording, when a " 1 " is intended and recorded correctly, and adding to it the expected probability of a " 1 " recording obtained through an incorrect recording of an intended " 0 ", response:

$$
p_{\text {exp }}=p_{\text {ogi }} \times\left(1-p_{\text {err }}\right)+\left(1-p_{\text {ogi }}\right) \times p_{\text {err }} .
$$

Since the log-likelihood function gives extremely high weight to errors that occur when pexp is close to zero or unity, the uncompensated method is overly sensitive to 
"outlier" responses. It was found that even with $p_{\mathrm{err}}$ set as low as .003 (chosen on the basis of the frequency of obviously wrong responses in the tail regions), the outlier sensitivity was culled and the fitted functions were intuitively in better agreement with the data plots. As a further bonus, no local minima problems appeared.

Goodness-of-fit measure. For quantal data analysis, there is no measure of fit that equals the $R^{2}$ of regular regression analysis. However, McFadden's $R^{2}$ (here called $R_{\mathrm{MF}}^{2}$ ) has some of the same characteristics (Amemiya, 1981; McFadden, 1974). It differs, for instance, by having a practical upper limit considerably lower than unity. This is due to the noise engendered by the quantal nature of the data in combination with the finite resolution entailed in the perceptual process. The $R_{\mathrm{MF}}^{2}$ can approach unity only if the threshold transition region falls entirely between two tested values on the independent variable. In a well-designed threshold experiment, this will of course not be the case; hence, the $R_{\mathrm{MF}}^{2}$ will always be less than unity-lower the more densely the independent variable is sampled relative to the observers' discriminative acuity for it. For comparing candidate invariants applied to the same data set, this should be acceptable, however.

No-constant regression. An important issue in regression analysis is whether a constant should be included in the model equation. A regression constant could often be understood as a response bias. In the present context, the bias would have to be in a consistent direction on the massratio variable, that is, relative to the impinging versus impinged-upon object distinction. This would require that the impinging object could be discerned as such on the basis of some trivial nonkinematic information alone. However, the advanced randomization of the display conditions precludes identification of the objects. The relevant interpretation of a significant regression constant is therefore that the observers are in fact not using information in accordance with the candidate invariant under test. Thus, a regression constant would represent an unknown factor influencing the judgments (the analysis only determines its magnitude). Yet, the goodness-of-fit measure would come out as if that factor were already known and part of the invariant. Including a constant in the model equation can only magnify the $R_{\mathrm{MF}}^{2}$, and hence will lead to a spurious increase in the fit of the tested invariantmore so for the poorer candidates. It follows that evaluation of candidate invariants should be done by means of no-constant analyses. Nonetheless, when attempting to define better candidate invariants, it could be useful to observe whether a better fit is obtained with a constant.

Each tested candidate invariant entails a comparison between the two objects on a postcollision kinematic property, defined in Figure 7, and was expressed either as a difference or as the logarithm of a ratio. It thus has an inherent zero point that potentially specifies the threshold and makes no-constant regression meaningful. In addition, it was scaled so that the most extreme value was either plus or minus unity (see Figures 8 and 9 below).

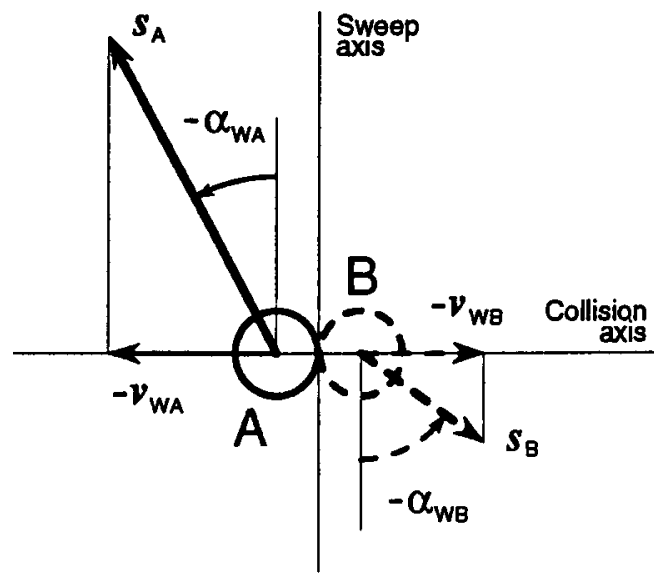

Figure 7. Kinematic properties used in defining candidate postcollision invariants. For each object, the velocity vector $s$ describes its motion after impact. The speed property is defined as the magnitude of $s . v_{w}$ is the collision-axis component of $s$ and $\alpha_{w}$ its angular deviation from the sweep axis. $v_{w}$ and $\alpha_{w}$ make up the win-velocity and the win-angle properties, with the signs chosen to reflect the outcome of the encounter between the objects. As captured by the win prefix, these properties are counted positive when the object proceeds across the sweep axis from where it is at impact. In the illustrated example, both objects "lose" to some extent in the collision; that is, they retreat after impact, so that the win measures are negative.

The names of the candidate invariants, given in Table 4, describe their composition.

The results are presented in Table 4 . The first row concerns regression of the empirical data on the criterion variable (mass ratio) and thus provides an index of the observers' performance. It confirms that performance drops radically when the precollision epoch is occluded, from a high level (indicated by the fact that a with-constant analysis does not raise the $R_{\mathrm{MF}}^{2}$ to more than .60) down to essentially nil (see also Figure 6). The following rows describe the fit of the candidate invariants, all based on properties available during the postcollision epoch. In the visible condition, all of them have a much poorer fit than the criterion, thus providing yet another confirmation that performance is better than the postcollision kinematics alone would allow. It follows that viable invariants for the visible condition will have to be assembled from precollision properties as well (to be treated in a forthcoming study).

In the occluded condition, on the other hand, the fit of all the candidate invariants surpasses that of the criterion, thus capturing, to varying degrees, what the judgments are based on in lieu of the full kinematics. The first two candidates are based on absolute postcollision speeds, whereas the following two are defined with reference to the collision-axis system (see Figure 7). The last pair are linear combinations, optimally weighted for the occludedcondition results. Clearly, the collision-axis-based invariants provide a better fit to the judgments than do the speed-based. No appreciable improvement is obtained by 
Table 4

Goodness-of-Fit (McFadden's $\boldsymbol{R}^{2}$ ) for Judgments

Obtained in Experiment 3, Regressed on

Criterion (Mass Ratio) and Candidate Invariants

\begin{tabular}{lcc} 
& $\begin{array}{c}\text { Normal } \\
\text { View }\end{array}$ & $\begin{array}{c}\text { Occluded } \\
\text { Precollision }\end{array}$ \\
\hline Mass ratio (log) & .57 & .10 \\
Speed ratio (log) & .10 & .25 \\
Speed difference & .16 & .32 \\
Win-angle difference & .23 & .39 \\
Win-velocity difference & .25 & .42 \\
Win-angle difference + speed difference $\times .39$ & .23 & .41 \\
Win-velocity difference + speed difference $\times .03$ & .25 & .42 \\
\hline
\end{tabular}

Note-All candidate invariants are defined on postcollision kinematics only. See Figure 7 for the definition of win angle and win velocity. Last two rows are optimal linear combinations for the occluded condition.

forming linear combinations between them. Further support for the adequacy of the win-velocity invariant is that if a constant is included in the model equation it comes out very close to zero and the $R_{\mathrm{MF}}^{2}$ is not improved. The same holds for power functions of the win-velocity difference. The best fit is achieved with the exponent set to unity. Also, performing the tests on the no-glitch data alone did not improve the $R_{\mathrm{MF}}^{2}$ values appreciably.

Further insight concerning the fit of the candidate invariants to the occluded condition results is provided by Figure 8. The scatterplots confirm the ranking of the candidates. They also demonstrate that the superiority of win velocity over win angle is larger than indicated by the $R_{\mathrm{MF}}^{2}$ values. The win-velocity crossover curve is steeper and has good fit also in the central region (where the $R_{\mathrm{MF}}^{2}$ measure is rather insensitive to deviations). One may view the experiment as a difference threshold measurement in which the effective stimulus variable is initially unknown and manipulated only indirectly. The win-velocity plot then stands out as having the obvious character of a threshold transition on a continuous stimulus variable. Taken together the evidence suggests, not only that win velocity is better than the other candidate invariants, but also that it truly is the property of the displayed events that is perceptually effective in judging the occluded collisions.

The informational characteristics of the postcollision candidate invariants-that is, their relations to the target property (mass ratio)-are illustrated in Figure 9. In each panel, the points fall along distinct curves for the four sweep $\times$ Vcomm combinations. The occurrence of such multiple mappings shows that the candidate property is not invariant under these circumstantial sources of variation. Win velocity has the advantage of being invariant under sweep variations; hence, there are only two curves.

Two other types of informational insufficiency are also illustrated: nonlinearities and offsets. It might seem that offsets (i.e., when the curves do not run through the origin) could easily be nulled in the perceptual process. Thus overall performance would be improved if the perceiver placed the PSE at a certain nonzero (i.e., negative) value on the invariant. Especially in the cue-heuristic perspective, biasing the judgments in this way should appear a simple feat in comparison with the extraction of velocity components projected on the abstract collision axis. However, despite the practice, the observers have clearly not done so (Figure 8 ). This confirms our reasoning behind the use of no-constant regression. There is no information in the postcollision epoch to distinguish the impinging from the impinged-upon object; hence, there is no way to bias judgments in a consistent direction.

Overall, win velocity exhibits the better specification power, whereas speed ratio has the poorest. This progres-
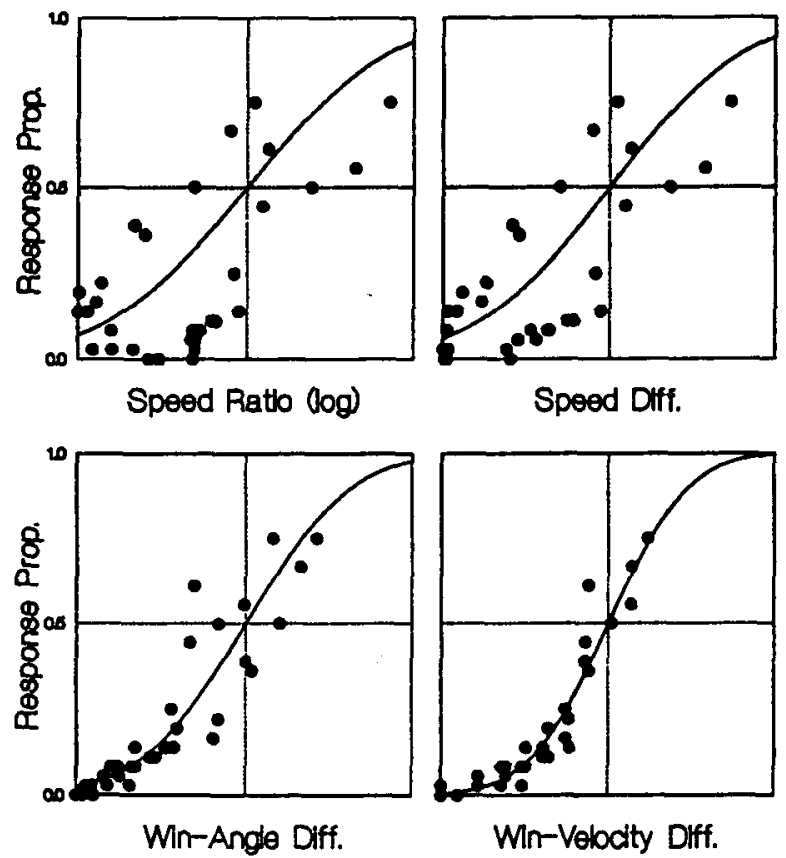

Figure 8. Results of Experiment 3, obtained with the precollision epoch occluded, plotted as a function of four candidate invariants. Fitted normal ogives also shown. Data are summed over the glitch, replications, and observers variables $(n=36$ for each plot point). Note that for win velocity the fit is somewhat better than it appears, because several of the points closest to the ogive are actually double. Moreover, the most deviant point (at prop $=.61$ ) is mainly due to the glitch manipulation. 


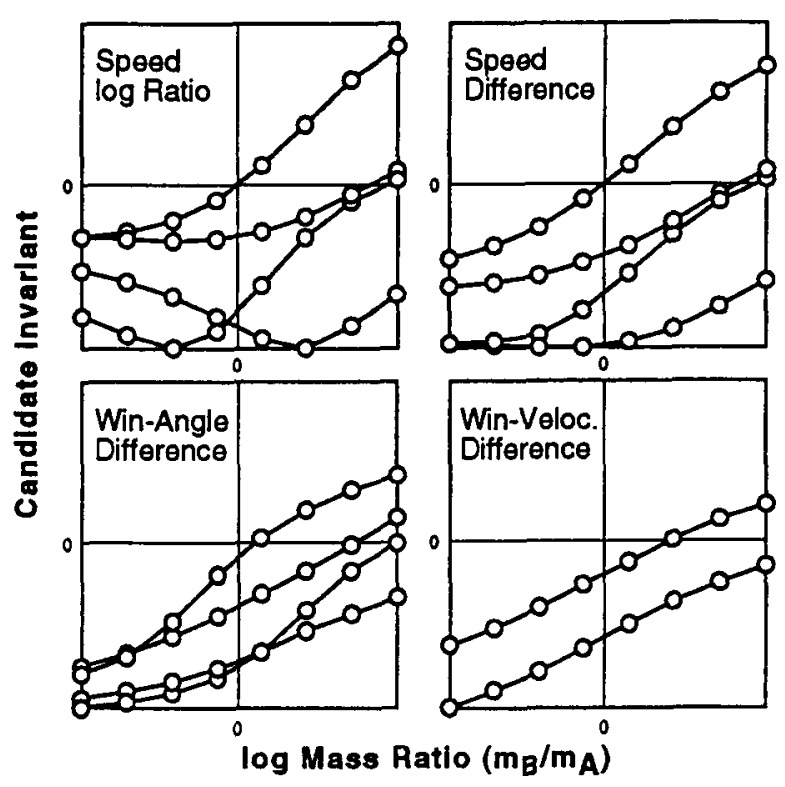

Figure 9. Candidate postcollision-only invariants plotted as functions of the criterion (log mass ratio). The diagrams illustrate insufficiencies (offsets, nonlinearities, and lack of invariance) of postcollision properties as specifiers of mass ratio. Lines connect points belonging to the same sweep $\times$ Vcomm conditions. Win velocity is invariant to sweep conditions; thus there are different lines for the two Vcomm conditions only.

sion coincides well with the order of the goodness-of-fit results shown in Table 4 and in Figure 8. Thus the candidate invariant that provides the best (i.e., least bad) postcollision-only information about mass ratio is also the one that best describes performance in the occluded condition. In other words, when barred from the essential precollision epoch, the observers manage to get the best thing possible out of the remains. ${ }^{10}$

\section{GENERAL DISCUSSION}

In several ways, the present study has shown that in the perception of relative mass through the observation of colliding objects, the effective information extends across both the precollision and the postcollision epochs of the event. Indeed, the level of performance attainable by human observers on this task has proven to be high enough that attempts at explaining perceptual functioning on the basis of momentary elemental cues are futile. The more viable approach is to treat the effective information as an analytically complex relation among pre- and postcollision kinematic properties. Thus it will have the form of an informative higher order property (invariant), available over time, in the Gibsonian ecological sense. A property of a proximal pattern identified in this way will be as real as any of the elemental properties into which it can be analytically decomposed; hence, it is potentially no less amenable to direct pickup (Runeson, 1977, 1992). The specifics of this information will be treated in a separate study.
For the assessment of human capabilities to perceive dynamic properties through vision, it should now be clear that occluded precollision events-because they do not allow the pertinent perceptual skills to come to the foremust be dismissed as abnormal and irrelevant. Nevertheless, the present occluded-precollision results have brought evidence on fundamental characteristics of visual functioning and thus have a bearing on the contrast between cue-based and invariant-based approaches to perception theory. It would therefore have been interesting if Gilden and Proffitt's (1989) cue-heuristic model could have been expressed as a quantitative variable and tested along with the candidate invariants put forth in the present study. This does not appear to be possible, however. Although that model's first cue is equivalent to speed ratio or speed difference, the second cue ("scatter") is defined, for both objects, with reference to the direction of the "incoming" object. This may make sense for collisions with a stationary object, but it is obviously ill-suited when both objects are initially in motion. Moreover, the incoming direction of neither object is available in the postcollision epoch, and hence the scatter cue is not a proper constituent of Gilden and Proffitt's model anyway-given their postulate that all cues must be simultaneously available. ${ }^{11}$ The model additionally requires salience criteria for determining which of the two cues is to be relied on in the individual cases. Since the criterion for scatter salience is formulated with reference to the same incoming direction as that of the cue itself (i.e., scatter angle $>90^{\circ}$ ), the objections above apply yet again.

However, Gilden and Proffitt's (1989) scatter cue does have some affinity with win angle, the second best invariant tested in the present study. Both concern the directions of motion after impact. Moreover, we now know, empirically at least, that the win-angle difference is available without access to the precollision epoch. Thus, if scatter is replaced with win angle, their model might be made to fit the current occluded-condition data quite well (if one assumes that salience could be redefined to allow win angle to dominate).

There are two reasons why Gilden and Proffitt's (1989) model could not be salvaged in this way. First, win angle is defined relative to the abstract collision-axis system; hence, including it would violate the second premise of the model-that it should comprise elemental nonderived cues only (see also below). Second, although Gilden and Proffitt claim universal validity for their model, the revision would only make it fit judgments obtained in the aberrant condition of occluded precollision. As we have seen, none of the postcollision properties has an appreciable predictive value in the visible condition (Table 4).

The prospects for adapting Gilden and Proffitt's (1989) model to normally visible collisions are even more discouraging, given the high level of the attainable performance. One would have to increase, very substantially, the already large number of constituents of the model. For instance, it would be necessary to include new cues pertaining to the precollision motions (thus giving up the simultaneity postulate), new salience criteria, and new 
common-sense notions about how they all operate together. Thus, the model would be exceedingly unparsimonious. This is in contrast with the simplicity that characterizes an invariant-based explanation as a result of its reliance on sophisticated, and hence more powerful, informative properties.

In addition to invalidating Gilden and Proffitt's (1989) primary claim as to the perceptual irrelevance of the precollision epoch, the results of the present study also undercut the second premise for their cue-heuristic approach. Although the authors acknowledge the superior informational potential of motion vector components, they argue that "observers do not derive these projections" and that "instead they treat the ... velocities along the trajectories and the angle ... as separate categories of information"' (p. 382). The model therefore describes how perceivers use either one or the other of these elemental properties at a time, as determined by mediational rules. However, our Experiment 3 (Table 4; Figure 8) has shown that collision-axis-based invariants have a better fit to the obtained judgments than those based on trajectory speeds, with the best $R_{\mathrm{MF}}^{2}$ occurring for the candidate that is exclusively based on collision-axis projections. Thus the empirical fact is that human performance on the task is in the best accordance with precisely the kind of properties dismissed by Gilden and Proffitt as fundamentally inaccessible. The present evidence for this comes especially from collisions with the precollision epoch occluded-a condition that might have been expected to facilitate cue-based judgments because of its relative paucity of kinematic elements.

It is worth noting that Gilden and Proffitt's (1989) denial of the perceptual role of motion vector components stands in sharp contrast with the results obtained by Gunnar Johansson in his pioneering studies of planar motion configurations and motions in depth (Johansson, 1950, 1964) and in subsequent studies by other authors (reviewed in Johansson, von Hofsten, \& Jansson, 1980). The finding common to these studies is that perception is fundamentally based on velocity vector components of the motions of the elements in the display. The present results verify the relevance of this notion for the perception of dynamic events as well.

Finally, Gilden and Proffitt's (1989) failure to reveal the indispensability of the precollision epoch and the advanced nature of the effective perceptual information is obviously a result of their having studied only a special case of collision conditions. It is instructive to see how spurious support for an elementaristic approach can arise as an artifact of the use of an experimental design that is lacking in crucial aspects of ecological relevance (cf. Burton \& Turvey, 1990; Rosen, 1978). An experiment cannot yield evidence about a mode of perceptual functioning that is more advanced than the sophistication entailed by the task and the experimental design and analysis.

\section{REFERENCES}

AmemiYA, T. (1981). Qualitative response models: A survey. Journal of Economic Literature, 19, 1483-1536.
BINGHaM, G. P. (1987). Kinematic form and scaling: Further investigations on the visual perception of lifted weight. Journal of Experimental Psychology: Human Perception \& Performance, 13, 155-177.

Bingham, G. P. (1993). Scaling judgments of lifted weight: Lifter size and the role of the standard. Ecological Psychology, 5, 31-64.

Burton, G., \& Turvey, M. T. (1990). Perceiving the lengths of rods that are held but not wielded. Ecological Psychology, 2, 295-324.

Finney, D. J. (1971). Probit analysis (3rd ed.). Cambridge: Cambridge University Press.

GiLDEN, D. L. (1991). On the origin of dynamical awareness. Psychological Review, 98, 554-568.

Gilden, D. L., \& Proffitt, D. R. (1989). Understanding collision dynamics. Journal of Experimental Psychology: Human Perception \& Performance, 15, 372-383.

GulLFORD, J. P. (1954). Psychometric methods. New York: McGraw-Hill. Johansson, G. (1950). Configurations in event perception. Uppsala, Sweden: Almqvist \& Wiksell.

Johansson, G. (1964). Perception of motion and changing form. Scandinavian Journal of Psychology, 5, 181-208.

Johansson, G., von Hofsten, C., \& Jansson, G. (1980). Event perception. Annual Review of Psychology, 31, 27-63.

Kaiser, M. K., \& Proffitt, D. R. (1987). Observers' sensitivity to dynamic anomalies in collisions. Perception \& Psychophysics, 42, 275-280.

MCFADDEN, D. (1974). Conditional logit analysis of qualitative choice behavior. In P. Zarembka (Ed.), Frontiers in econometrics (pp. 105142). New York: Academic Press.

Meriam, J. L. (1975), Dynamics (2nd ed.). New York: Wiley.

MichotTE, A. (1963). The perception of causality (T. R. Miles, Trans.). London: Methuen. (Original work published 1946)

Press, W. H., Flannery, B. P., Teukolsky, S. A., \& Vetterling, W. T. (1986). Numerical recipes. Cambridge: Cambridge University Press.

Proffitt, D. R., \& Gilden, D. L. (1989). Understanding natural dynamics. Journal of Experimental Psychology: Human Perception \& Performance, 15, 384-393.

ROSEN, R. (1978). Fundamentals of measurement and representation of natural systems. New York: North-Holland.

Runeson, S. (1974). Constant velocity-Not perceived as such. Psychological Research, 37, 3-23.

RUNESON, S. (1975). Visual prediction of collision with natural and nonnatural motion functions. Perception \& Psychophysics, 18, 261-266.

RuNEson, S. (1977). On the possibility of 'smart' perceptual mechanisms. Scandinavian Journal of Psychology, 18, 172-179.

RunEson, S. (1983). On visual perception of dynamic events. (Acta Universitatis Upsaliensis: Studia Psychologica Upsaliensia, Serial No. 9). (Original work published 1977)

RunEson, S. (1989a, July). An invariant-based model for the acquisition of perceptual skills. Paper presented at the Fifth International Conference on Event Perception and Action, Miami University, Oxford, $\mathrm{OH}$.

Runeson, S. (1989b). A note on the utility of ecologically incomplete invariants. International Society for Ecological Psychology Newsletter, 4(1), 6-9.

RUNESON, S. (1992). Psychophysics: The failure of an elementaristic dream. Manuscript submitted for publication.

Runeson, S. (in press). Perception of biological motion: The KSD-principle and the implications of a distal versus proximal approach. In G. Jansson \& S. S. Bergström (Eds,), Perceiving events and objects: A review of Gunnar Johansson's research with commentaries.

Runeson, S., \& Costall, A. P. (1991). The ecological concept of information versus the traditional notion of cue. Unpublished manuscript.

Runeson, S., \& Frykholm, G. (1981). Visual perception of lifted weight. Joumal of Experimental Psychology: Human Perception \& Performance, 7, 733-740.

Runeson, S., \& Frykholm, G. (1983). Kinematic specification of dynamics as an informational basis for person and action perception: Expectation, gender recognition, and deceptive intention. Journal of Experimental Psychology: General, 112, 585-615.

Thinès, G., Costall, A. P., Butterworth, G. (Eds.) (1991). Michotte's experimental phenomenology of perception. Hillsdale, $\mathrm{NJ}$ : Erlbaum. 
TIPLER, P. A. (1976). Physics. New York: Worth.

TODD, J. T., \& WARREN, W. H. (1982). Visual perception of relative mass in dynamic events. Perception, 11, 325-335.

WARREN, W. H., KIM, E. E., \& HUSNEY, R. (1987). The way the ball bounces: Visual and auditory perception of elasticity and control of the bounce pass. Perception, 16, 309-336.

\section{NOTES}

1. The KSD principle is misrepresented in some recent works (Gilden, 1991; Gilden \& Proffitt, 1989). Despite explicit statements to the contrary (Runeson, 1977/1983; Runeson \& Frykholm, 1983), KSD is taken to be a comprehensive theory of perception rather than an identification of available support for it. Furthermore, the fictitious theory attributed to the KSD label features the nature of perception in a way that hardly has any proponents.

2. In the present paper, the term collision axis is used in reference to the direction of the impact forces. Thus, it is the dimension in which all exchange of momentum between the two objects occurs (cf. Meriam, 1975). As a good approximation, for homogeneous circular objects, the collision axis runs through the centers of the two objects at the moment of (peak) impact (cf. Figures 2 and 7). It should be observed that Gilden and Proffitt (1989, e.g., p. 374; Gilden, 1991) use "collision axis", differently, referring to the direction of motion of the incoming object.

3. The present study is focused on the empirical side and on the role of the precollision epoch. A detailed treatment of the availability of information in the kinematics of collisions and a critique of Gilden and Proffitt's (1989) Experiment 2 are deferred for a forthcoming paper.

4. In Gilden and Proffitt's (1989) experiments, as well as the ones reported in the present study, simulated displays were used instead of real colliding objects. For this reason, it is not possible to assign absolute values of mass to the two objects; only relative mass can be meaningfully controlled. It follows that it is not possible to distinguish a standard and a variable stimulus. Since the selection, placement, and presentation order of the standard have proven to be intricate sources of bias, the present type of experiment provides an interestingly pure variety of the method of constant stimuli.

5. The simulation is valid for objects that slide without spin in the manner of hockey pucks, rather than roll like balls. Moreover, friction between the objects is not simulated, and hence, spin does not arise in the collisions.

6. The mechanics of collisions is essentially indifferent to all forces except those generated by the impact itself (Meriam, 1975, p. 152; Tipler, 1976, p. 281). However, in the simulation, the forces producing the lead-in motions were applied only up to the moment of contact between the objects; hence, the objects continued without acceleration or deceleration after the collision.

7. Gilden and Proffitt's (1989) simulation employed no dissipation. Hence, their collisions had perfect elasticity (restitution $=1.0$ ), which cannot occur naturally.

8. We are using speed to designate an object's velocity along its trajectory, as distinguished from velocity, which we use for vector components projected on the collision or the sweep axis.

9. Alternatively, the term local invariant is used instead of incomplete invariant, because such a property will typically be fully valid within some restricted domain of conditions (Runeson \& Costall, 1991).

10. Because practice with feedback was given before the occluded blocks, there is, in principle, a risk that observers may have recognized individual experimental conditions and remembered the correct responses. This possibility was precluded by the rich random variation in the display conditions. Moreover, the judgments did not agree with the criterion. Instead they adhered to the win-velocity property, on which feedback was not given.

11. This insight now appears to be shared by one of the authors (Gilden, 1991, p. 562).

(Manuscript received January 6, 1992; revision accepted for publication November 10, 1992.) 